\title{
Reduced-Order Modeling of Hypersonic Unsteady Aerodynamics Due to
}

\section{Multi-Modal Oscillations}

\author{
Torstens Skujins * \\ Carlos E. S. Cesnik ${ }^{\dagger}$ \\ University of Michigan, Ann Arbor, Michigan 48109
}

\begin{abstract}
The development of hypersonic vehicle control algorithms requires the accurate prediction of vehicle aerodynamic loads. This paper focuses on finding time-accurate unsteady loads due to the oscillation of multiple vehicle elastic modes. The reduced-order modeling methodology consists of using convolution of modal step responses along with a correction factor to take different oscillation amplitudes, flight conditions, and nonlinear aerodynamic effects due to multi-modal oscillations into account. Thus, the model is valid over a range of conditions rather than one specific set of conditions upon which it is constructed. When compared with CFD simulations, the errors are shown to be relatively small in most cases over a range of Mach numbers, modal frequencies, and modal oscillation amplitudes. Also, an error estimation methodology is developed to give a general sense beforehand as to the errors expected to be incurred through the use of the model.
\end{abstract}

\section{Introduction}

Among the many challenges faced in the design of hypersonic vehicles is the development of accurate control algorithms. The aerodynamic forces in the hypersonic regime are quite large, so any misprediction of these loads will potentially result in inaccurate control algorithms, which in turn could result in loss of control of the vehicle. Even though hypersonic vehicles are inherently stiff structures, they still do experience flexible deformations while in flight. Thus, to accurately model the aerodynamic loads on the vehicle, it is important to create the tools to accurately model the unsteady aerodynamic effects caused both by these structural deformations as well as rigid body pitch/plunge motions.

Computational fluid dynamics (CFD)-based reduced-order models provide an effective way to model the unsteady aerodynamic loads. Once constructed, the models run orders of magnitude faster than full CFD solutions while preserving a high level of the accuracy seen by the computational simulations. However, a major drawback of many reduced-order models is that they are only valid for flight conditions immediately around those from which the model was constructed. Efforts have been made to make the ROMs valid over a range of parameters. Glaz et al. ${ }^{1}$ use an unsteady surrogate-based approach to construct a model for unsteady rotorcraft dynamics over a range of pitch/plunge motions and Mach numbers. Silva ${ }^{2}$ uses a convolution-type of methodology to construct a state-space ROM which is then used over a range of velocities in the transonic regime by modifying the time step of the numerical integration. Other efforts for parameterindependent ROMs have focused on the analysis of flight test data. Lind et al. ${ }^{3}$ create velocity-independent kernels by using curve fits of flight test data gathered at different conditions. Baldelli et al. ${ }^{4}$ create a model valid over a range of dynamic pressures by combining linear and nonlinear operators for model construction. Prazenica et al. ${ }^{5}$ extrapolate kernels found at different flight conditions to create one model valid over a range of conditions. Omran and Newman ${ }^{6}$ use Volterra series submodels in different domains, such as pre-stall and post-stall, to construct an overall global piecewise Volterra series model.

\footnotetext{
${ }^{*}$ Graduate Research Assistant, University of Michigan, Email: tskujins@umich.edu, Member AIAA.

$\dagger$ Professor, Department of Aerospace Engineering, University of Michigan, Email: cesnik@umich.edu, 1320 Beal Avenue, 3024 FXB, Ann Arbor, MI 48109-2140, Ph. (734) 764-3397, Fax: (734) 764-0578, AIAA Associate Fellow.
} 
Ref. 7 lays out the basic framework for a reduced-order model (ROM) technique for the hypersonic vehicle unsteady aerodynamics. The methodology is based on the convolution of modal step responses combined with a nonlinear correction factor to account for amplitudes of motion and flight conditions away from those around which the ROM was constructed. However, as detailed in the paper, the method was limited to cases with only a single mode of oscillation. This work extends this formulation to take into account multimodal oscillations and provides an assessment of the effects of reduced frequency on the accuracy of the model. In order to construct the nonlinear correction factor, a certain number of CFD sampling runs must be completed. This work also lays out a quantitative metric for determining an optimal number of sampling points to use and provides an estimate of the errors expected to be incurred through the use of the ROM methodology.

\section{ROM Methodology}

A convolution-based ROM was chosen due to the ease of implementation into a CFD code. In general, the process involves convolving the modal step response with arbitrary modal motion to find the uncorrected ROM response. Then, the nonlinear correction factor is applied to give the final corrected ROM response. The overall unsteady aerodynamic ROM framework is shown in Fig. 1. The inputs are the structural mode shapes and modal amplitudes at each time step, from which the modal motion can be obtained. The outputs are the time-accurate coefficients and/or generalized aerodynamic forces. See Ref. 7 for details on the CFD runs necessary for model construction.

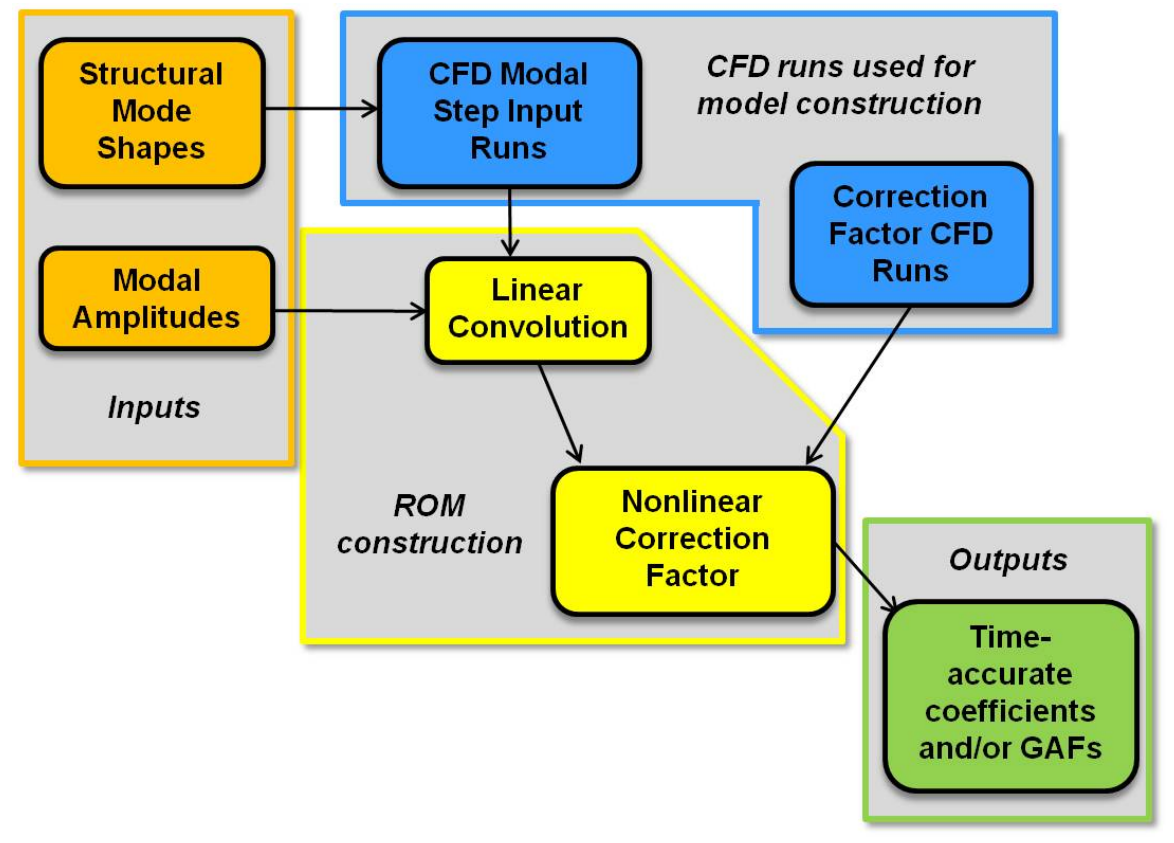

Figure 1. Unsteady aerodynamic ROM schematic

\section{A. Convolution}

The response of a linear system to an arbitrary input can be found if the response of the system to a unit step $(H(t))$ or unit impulse $(h(t))$ function is known. The response $y(t)$ due to an arbitrary input $f(t)$ is found through the use of convolution: ${ }^{8,9}$

$$
y(t)=f(0) H(t)+\int_{0}^{t} \frac{d f}{d t}(\tau) H(t-\tau) d \tau
$$

Since the unit impulse is the derivative of the unit step, integration by parts yields 


$$
y(t)=f(t) H(0)+\int_{0}^{t} f(\tau) h(t-\tau) d \tau
$$

Equations 1 and 2 are the two forms of Duhamel's integral.

The reduced-order model presented here is based on the step convolution described above. Volterra series is the nonlinear extension of convolution. However, for the cases tested here, the ROM using convolution and the correction factor described subsequently showed improved agreement with direct CFD results when compared with Volterra results. The uncorrected ROM solution is found by convolving the arbitrary modal motion with CFD modal step input response results.

\section{B. Correction Factor, Single Mode}

The results in Ref. 7 showed that, as the input amplitude and flight conditions strayed from those used in the step response, the accuracy of the lift, drag, and moment coefficients significantly decreased. Thus, a nonlinear correction factor was introduced, defined as follows for the oscillation of a single mode:

$$
f_{c}=\frac{\frac{Y_{2}}{Y_{1}}}{\frac{d_{2}}{d_{1}}}
$$

In Eq. $3, Y_{1}$ is the final, quasi-steady response ( $c_{l}$ or other coefficient) to a step input of amplitude $d_{1}$, and $Y_{2}$ is the final response to an input of amplitude $d_{2}$, which may also be at a different flight condition. For a linear system, this ratio would always equal 1 , as the outputs scale with the inputs.

Latin hypercube testing combined with kriging methodology was used to construct surfaces for $f_{c}$ pertaining to each of the coefficients throughout the parameter space of interest. Results showed that multiplying the convolved response with this correction factor greatly improved the accuracy of the reduced-order model.

\section{Kriging}

Kriging is a methodology which creates surface fits of functions using sampling data points obtained through computational experiments. Unlike actual experiments, which inherently contain random error, computational simulations will give the same answer for the same simulation when repeated. Thus, a kriging surface will pass through all test points, whereas a least squares fit surface will not in general directly pass through each point. This is accomplished through the use of a regression model coupled with a random function. Refs. 10 and 11 provide detailed explanations of the kriging methodology and model construction.

\section{Correction Factor, Multiple Modes}

The extension of the correction factor to multiple modes is investigated at first using two different methods. Consider a sample airfoil undergoing oscillations in each of the first $m$ modes. The first method is to simply use superposition of individual modal responses to find the total response. Using this method, the response of each mode is calculated as detailed above. Then, each response is added together to find the final response.

The second method is to create a correction factor that can be easily extended to multiple modes. This can be accomplished by defining the multi-modal correction factor $f_{c m}$ as follows, here presented for simplicity as a three-mode excitation:

$$
f_{c m}=\frac{Y_{123}-\delta}{Y_{1}+Y_{2}+Y_{3}-\delta}
$$

In Eq. 4, $Y_{123}$ is the final quasi-steady response after steps of certain amplitudes have been simultaneously applied to each of the first three modes; the denominator is the superposition of uncorrected individual modal responses $Y_{1}, Y_{2}$, and $Y_{3}$; and $\delta$ is an offset introduced to prevent numerical issues. A kriging surface is created by finding correction factor values at sampling points throughout the parameter space; in this case, the variables are Mach number and modal amplitudes. Then, to apply the correction factor, the motion of each of the modes is individually convolved with the step response and added together via superposition to find the uncorrected response $Y_{u}$. Rearranging Eq. 4, the final corrected response value $Y_{c}$ is found as follows:

$$
Y_{c}=f_{c m}\left(Y_{u}-\delta\right)+\delta
$$


Note that a separate kriging surface is found for each of the coefficients of interest.

\section{E. Basic Problem Definition and Setup}

The CFD code used in this study is CFL3Dv6, developed at NASA Langley. ${ }^{12}$ The code is capable of solving the Euler/Navier-Stokes equations for both steady and unsteady flows on two and three-dimensional structured grids and has mesh deformation capability. Grids are created using the mesh generator ICEM CFD from ANSYS. ${ }^{13}$ All results shown are Euler solutions. Modal inputs are given to the airfoil geometry, described below, by utilizing the code's mesh deformation capabilities. Response quantities tracked are the lift, drag, and moment coefficients.

\section{Geometry}

The geometry used is a two-dimensional half diamond airfoil with a flat top surface. It is $2.5 \%$ thick and has a length of $1.6 \mathrm{~m}$. This is not intended to be representative of any specific airfoil or vehicle, as the method is general and can be applied to different configurations. The grid, shown in Fig. 2 (zoomed in on the airfoil) is a $548 \times 674$ structured grid with points concentrated more closely near the airfoil surface. The first mode step response obtained is virtually indistinguishable to that from a more refined grid of $644 \times 866$ points.

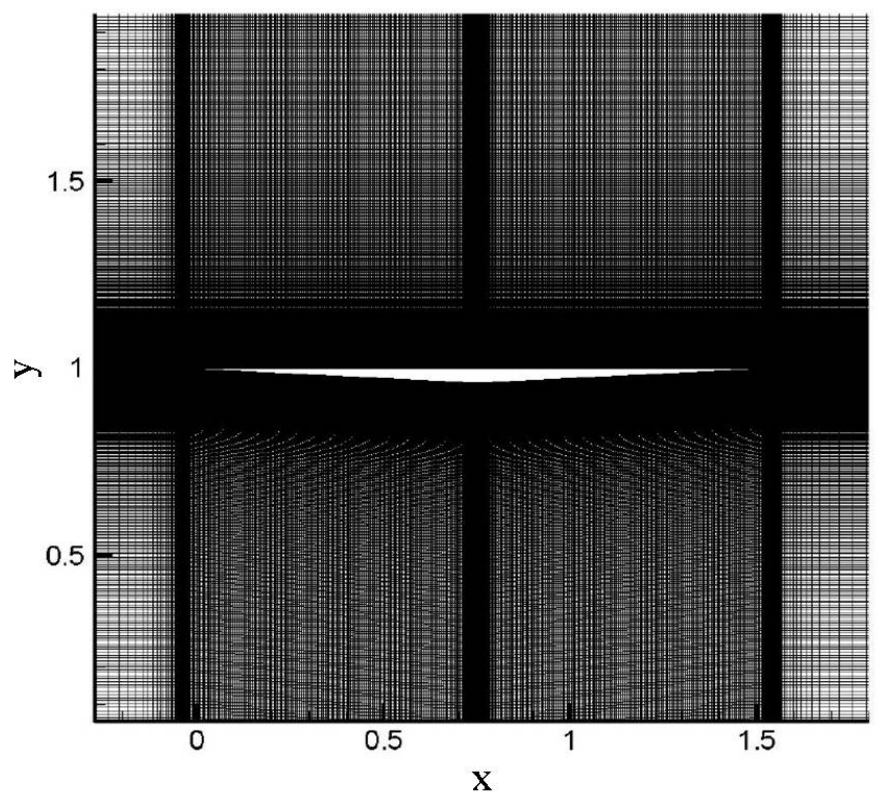

Figure 2. CFD half-diamond airfoil grid

\section{Mode Shapes}

Some fundamental deformation modes of the elastic structure must be used when creating the unsteady aerodynamic ROM. Typically, those fundamental modes are elastic mode shapes of the structure, and they would come from the solution of the structural dynamics part of the problem. To simulate those in our present study, three chordwise mode shapes were assumed. Like the geometry itself, the mode shapes assumed here do not correspond to any specific configuration. Figure 3 shows a plot of the centerline displacements of these mode shapes; the amplitudes shown correspond to those used for the step inputs.

\section{F. Error Metric}

The error metric used to judge the accuracy of the ROM is described as follows. At each time step, the ROM and CFD response values are compared, as shown in Eq. 6 with the various CFD quantities defined 


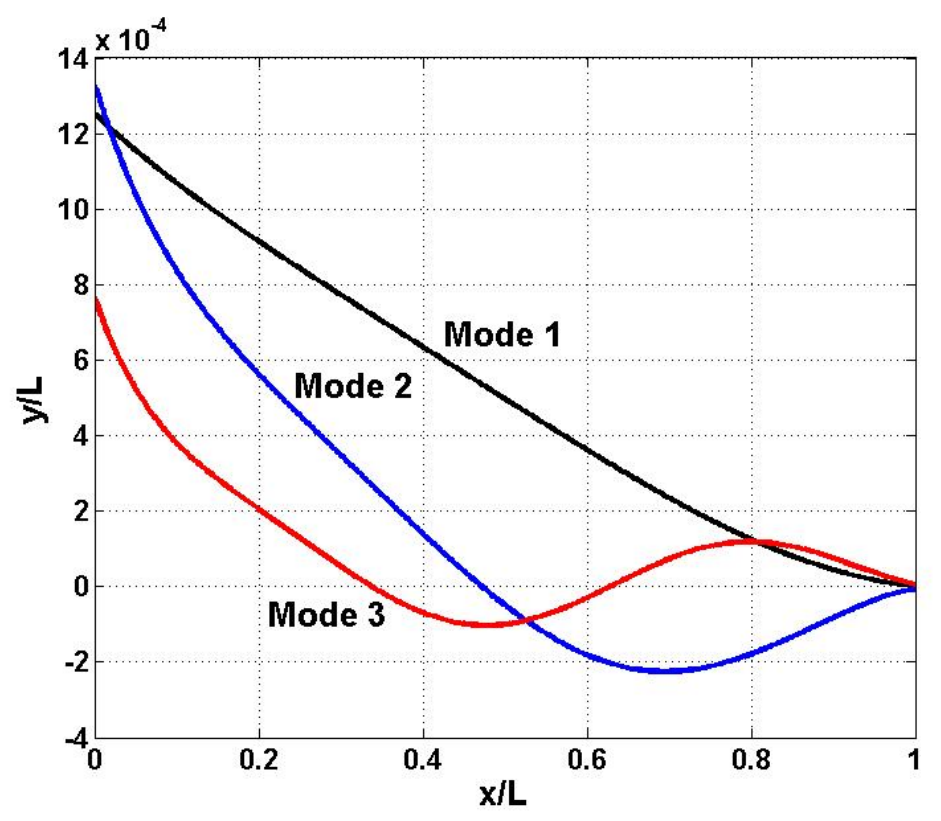

Figure 3. Mode shapes

in Fig. $4 ; i$ ranges from 1 to the final time step considered.

$$
\text { error }=\max \left(\frac{\left|C F D_{i}-R O M_{i}\right|}{C F D_{\max }-C F D_{\min }}\right) \times 100 \%
$$

\section{Reduced Frequency Studies}

Due to the high flow velocity, hypersonic vehicles tend to have low values of reduced frequency $k$, defined in Eq. 7,

$$
k=\frac{b \omega}{U_{\infty}}
$$

where $b$ is the airfoil's half-chord, $\omega$ is the frequency of oscillation, and $U_{\infty}$ is the freestream velocity. Since reduced frequency is an key determining factor of the flow's unsteadiness, it is important to investigate how the ROM is affected by increasing $k$ values.

Results showing the errors with increasing reduced frequency have been obtained for individual excitations of each of the first and third modes for the two-dimensional half-diamond airfoil. Figures 5(a) and 5(b) show the results for each mode for situations in which all flight conditions and modal amplitudes remain the same, but the reduced frequency, by changing the oscillation frequency, is modified on each CFD run. Though the errors do increase with $k$, they remain relatively small over the range tested, which corresponds to oscillation frequencies ranging from $125 \mathrm{rad} / \mathrm{s}$ to $1250 \mathrm{rad} / \mathrm{s}$. These two cases are run at Mach 8, which is the same Mach number as the step response upon which the ROM is based (i.e. the modal step of amplitude $d_{1}$, and hence response $Y_{1}$, in Eq. 3 are found at Mach 8).

Next, consider a case similar to that in Fig. 5 but with the Mach number different than that of the base step response. Figure 6 shows the errors a first mode case at Mach 5 and amplitude $33 \times$. The solid lines represent the errors if the Mach 8 step was used as the base ( $d_{1}$ and $Y_{1}$ from Eq. 3), while the dashed lines represent the errors if the base is a Mach 5 step response. The plot shows that the errors for the Mach 5 base are smaller than the Mach 8 base. Once a new CFD step response has been obtained for a specific flight condition, it does not take any extra computational time to use this new response in the ROM. Thus, it will be beneficial to have several different step responses available to use and to pick the one with flight 


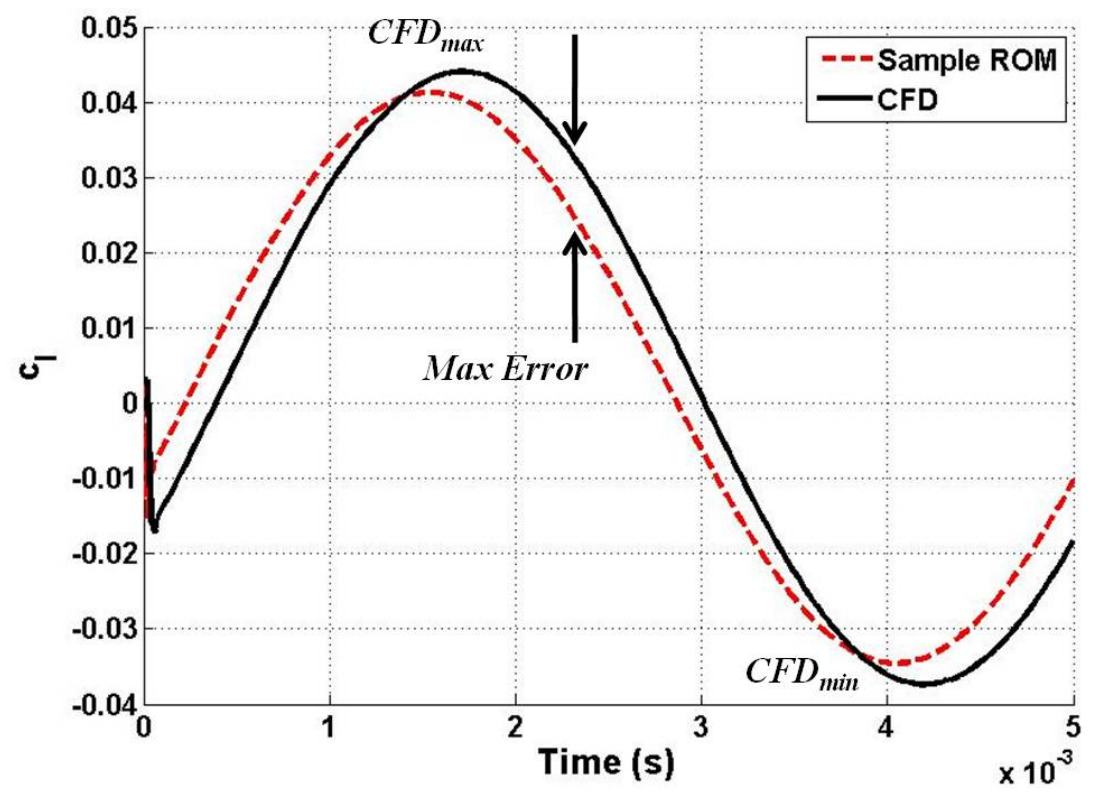

Figure 4. ROM-CFD comparison error metric

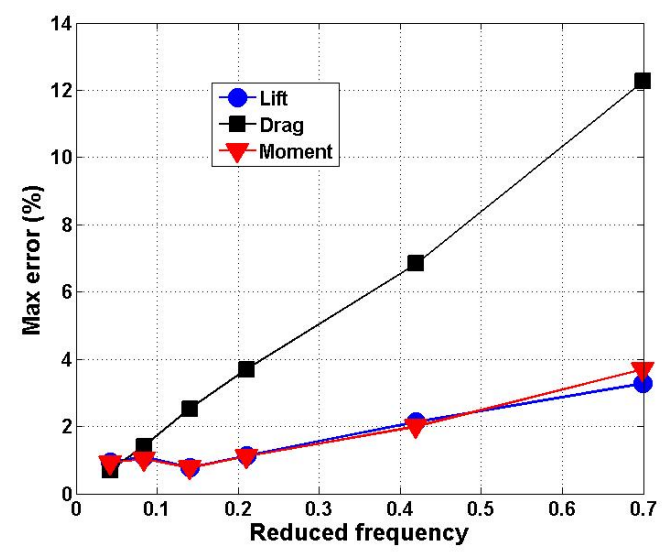

(a) Mode 1, amplitude $=40 \times$

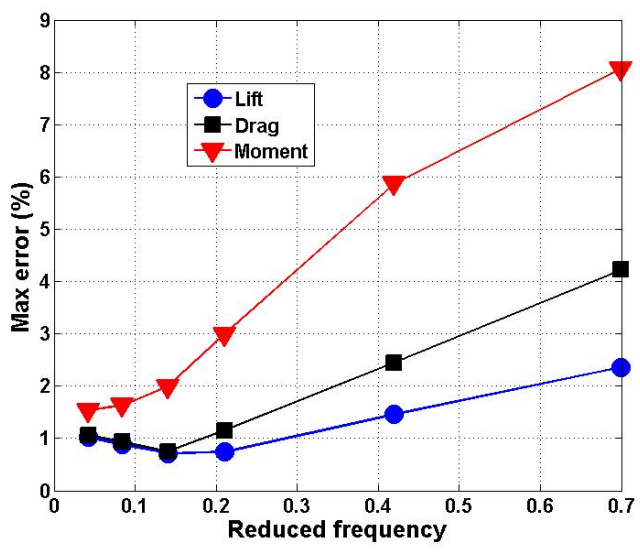

(b) Mode 3, amplitude $=10 \times$

Figure 5. Reduced frequency errors, Mach 8 
conditions closest to those in the simulation. Other than the step responses themselves, no additional CFD runs will be necessary, as the correction factor can be based on any arbitrary step response.

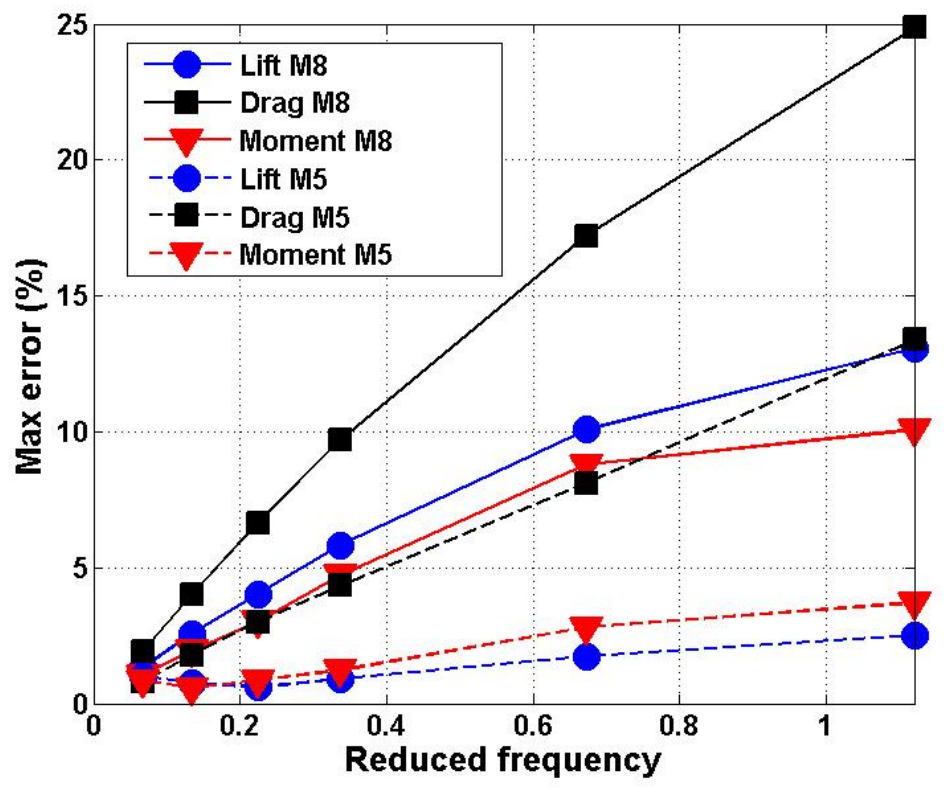

Figure 6. Mode 1, Mach 5, amplitude $=33 \times$

\section{Multi-Modal Oscillations}

To test the multi-modal correction factor, test cases with sinusoidal oscillations of each of the first three modes were conducted. Latin hypercube sampling ${ }^{14}$ was used to pick the parameter values, which consisted of the Mach number, modal amplitudes, and modal oscillation frequencies, for each run. Table 1 shows the range of values used as well as the values of the specific test case shown below. Note that the reduced frequency range corresponds to a modal oscillation frequency range of $100 \mathrm{rad} / \mathrm{s}$ to $1,000 \mathrm{rad} / \mathrm{s}$.

Table 1. Multi-modal oscillation parameter values

\begin{tabular}{cccc}
\hline \hline Parameter & Max & Min & Example \\
\hline$M$ & 5.0 & 9.5 & 7 \\
$d_{1}$ & $-60 \times$ & $60 \times$ & $53 \times$ \\
$d_{2}$ & $-25 \times$ & $25 \times$ & $13 \times$ \\
$d_{3}$ & $-25 \times$ & $25 \times$ & $-19 \times$ \\
$k_{1}$ & 0.028 & 0.535 & 0.19 \\
$k_{2}$ & 0.028 & 0.535 & 0.38 \\
$k_{3}$ & 0.028 & 0.535 & 0.27 \\
\hline \hline
\end{tabular}

Overall, the ROM showed good agreement with the CFD over the range tested. Figs. 7 and 8 show the ROM-CFD comparisons for the example case described in the table. Additionally, the straight superposition of individual modal responses is plotted as well.

Table 2 summarizes the maximum errors seen for the example case mentioned above. For each coefficient, the ROM had less error than superposition. Though each captured the lift coefficient relatively well, superposition did not predict the peaks as well for the moment coefficient as the ROM and thus had a larger 


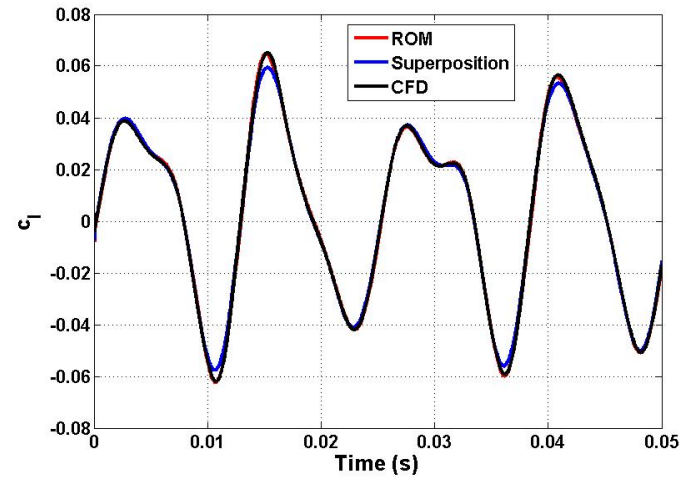

(a) Lift coefficient

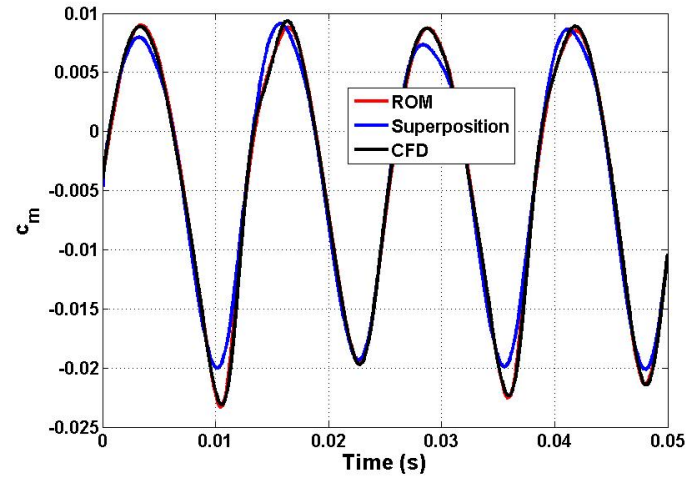

(b) Moment coefficient

Figure 7. ROM-CFD comparison, multi-modal oscillations

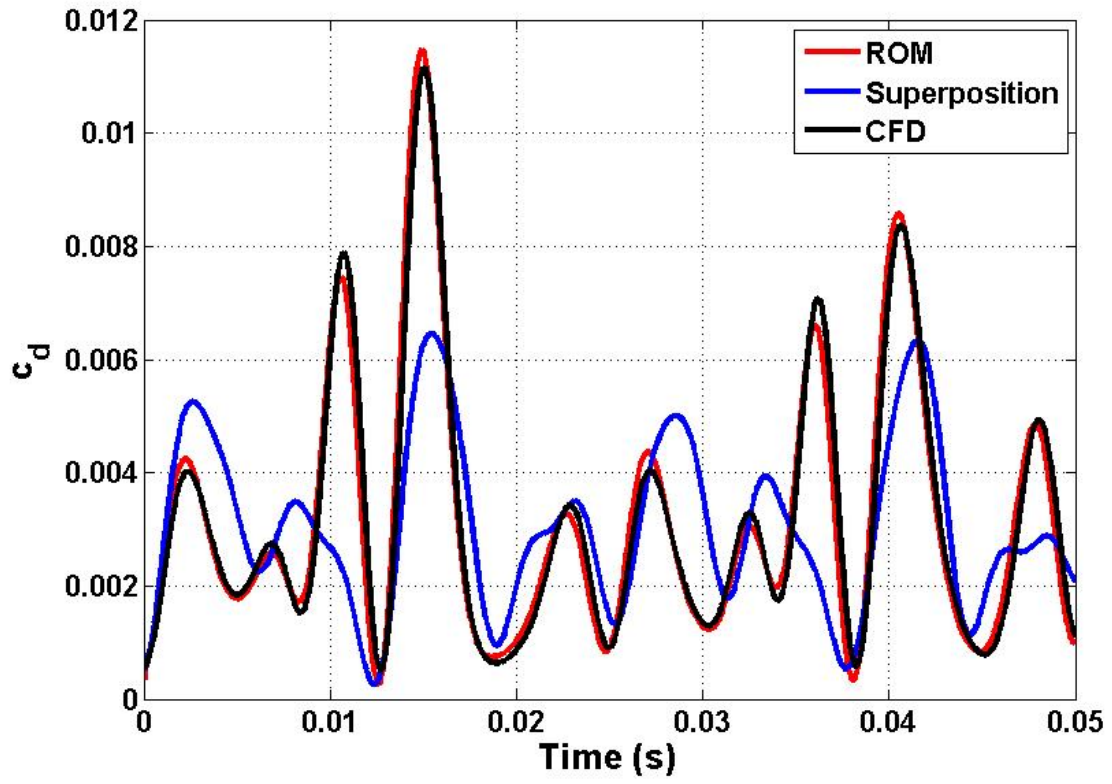

Figure 8. Drag coefficient 
error. For the drag coefficient, superposition is not close to the CFD results, as quantified by the over $50 \%$ error seen for that case. The ROM matches the CFD drag coefficient results well qualitatively; the errors seen are around the peaks of the plot.

Table 2. ROM and Superposition Errors (percent)

\begin{tabular}{ccc}
\hline \hline Coefficient & ROM error & Superposition error \\
\hline Lift & 1.78 & 4.51 \\
Moment & 3.28 & 12.86 \\
Drag & 10.04 & 53.79 \\
\hline \hline
\end{tabular}

Overall, the ROM matched the CFD results relatively well for each of the cases tested. However, this does not provide any sort of quantitative error estimate for the ROM methodology in general when applied to this configuration. Because of this, it is necessary to create an error estimation methodology give a more confident estimate of the errors expected to be incurred by the use of the ROM.

\section{Error Estimation}

When considering the overall error incurred by the ROM, two separate areas need to be considered. The first is the error of the kriging surface compared to the function it is modeling. Among the important issues faced when constructing the ROM is deciding on how many sampling points are needed for the correction factor kriging surface. Too few points would result in an inaccurate representation of the function and thus loss of accuracy of the ROM in general. However, using more points than necessary would result in unneeded computational expense. Thus, the first part of the error estimation focuses on finding the optimal number of sampling points to use. One method which assesses the error of kriging surfaces is the Efficient Global Optimization (EGO) algorithm. ${ }^{15,16}$ The purpose of this algorithm is to find global maxima and minima on surfaces; this is accomplished by placing points at locations of maximum expected improvement and uncertainty on the surface. The method presented here is similar to the EGO algorithm except for the fact that the purpose is to simply minimize the error on the surface, not to find the specific location of extrema. Thus, the addition of sampling points is based solely on the mean squared error of a location on the surface, not the likelihood of a new extrema being at a certain location.

The second area is the error of the function when compared with the truth model, thus far considered to be the CFD results. Even if the kriging surface matched the intended function exactly, the methodology would still result in some error. The second part of the error estimation focuses on quantifying this error.

\section{A. Error of Kriging Surface Compared to Function}

Due to the high computational expense of CFD simulations and thus kriging surface sample point calculations, it is important to know the number of points and location within the parameter space of each point before beginning model construction. Because of this, it is not feasible to use CFD itself to determine these items. Instead, some sort of simplified, computationally inexpensive models must be used. For this study, piston theory has been implemented.

Piston theory ${ }^{17,18}$ is a simplified method for calculating unsteady pressures on a supersonic body by using the approximation that a planar slab of fluid initially perpendicular to the flow direction will remain that way as it passes over a body. The normal velocity of the body surface may cause the slab to expand or compress as it travels down the surface, resulting in a changing pressure. Using the piston analogy, the pressure $p(x, t)$ on a point of the surface can be found by: ${ }^{19}$

$$
p(x, t)=p_{\infty}\left(1+\frac{\gamma-1}{2} \frac{v_{n}}{a_{\infty}}\right)^{\frac{2 \gamma}{\gamma-1}}
$$

In Eq. $8, p_{\infty}$ is the freestream pressure, $\gamma$ is the ratio of specific heats, $v_{n}$ is the velocity of the surface normal to the flow direction, and $a_{\infty}$ is the freestream speed of sound. Taking a third-order binomial expansion of 
the above expression, the third-order piston theory pressure at a certain location on the surface of the body is found as follows:

$$
p(x, t)=p_{\infty}+\gamma p_{\infty}\left[\frac{v_{n}}{a_{\infty}}+\frac{\gamma+1}{4}\left(\frac{v_{n}}{a_{\infty}}\right)^{2}+\frac{\gamma+1}{12}\left(\frac{v_{n}}{a_{\infty}}\right)^{3}\right]
$$

Note that piston theory breaks down when the normal velocity of the surface approaches the speed of sound as well as in areas where curvature introduces three-dimensional effects, as in a flow moving down a cylindrical body.

Figure 9 shows a diagram of this first error analysis section methodology. To start with, a beginning number of sampling points within the parameter space is selected using Latin hypercube sampling. At each sampling point, piston theory is used to calculate the final coefficient values instead of CFD. Then, using Eq. 4, these piston theory values are combined with CFD step responses to find the correction factor values at each of the points. Note that CFD is used for the step responses due to the relatively low computational cost involved, as the step response in general does not need to be at the same flight conditions as the sampling point in the parameter space, and thus only a small number of step responses will need to be calculated. Next, the kriging surface is constructed with the available sampling point data, and the maximum mean squared error (MSE) is calculated at points throughout the surface. This error $s^{2}$ at location $x^{*}$ in the parameter space is found as follows: ${ }^{15}$

$$
s^{2}\left(x^{*}\right)=\sigma^{2}\left[1-\mathbf{r}^{\prime} \mathbf{R}^{-1} \mathbf{r}+\frac{\left(1-\mathbf{1}^{\prime} \mathbf{R}^{-1} \mathbf{1}\right)^{2}}{\mathbf{1}^{\prime} \mathbf{R}^{-1} \mathbf{1}}\right]
$$

In Eq. 10, $\sigma$ is the surface's variance, $\mathbf{r}$ is a column of the correlation matrix $\mathbf{R}$, and $\mathbf{1}$ is a column vector of ones. See Refs. 10,11, and 15 for the derivation and a detailed explanation. In this work, this error calculation is obtained from a built-in MATLAB subroutine.

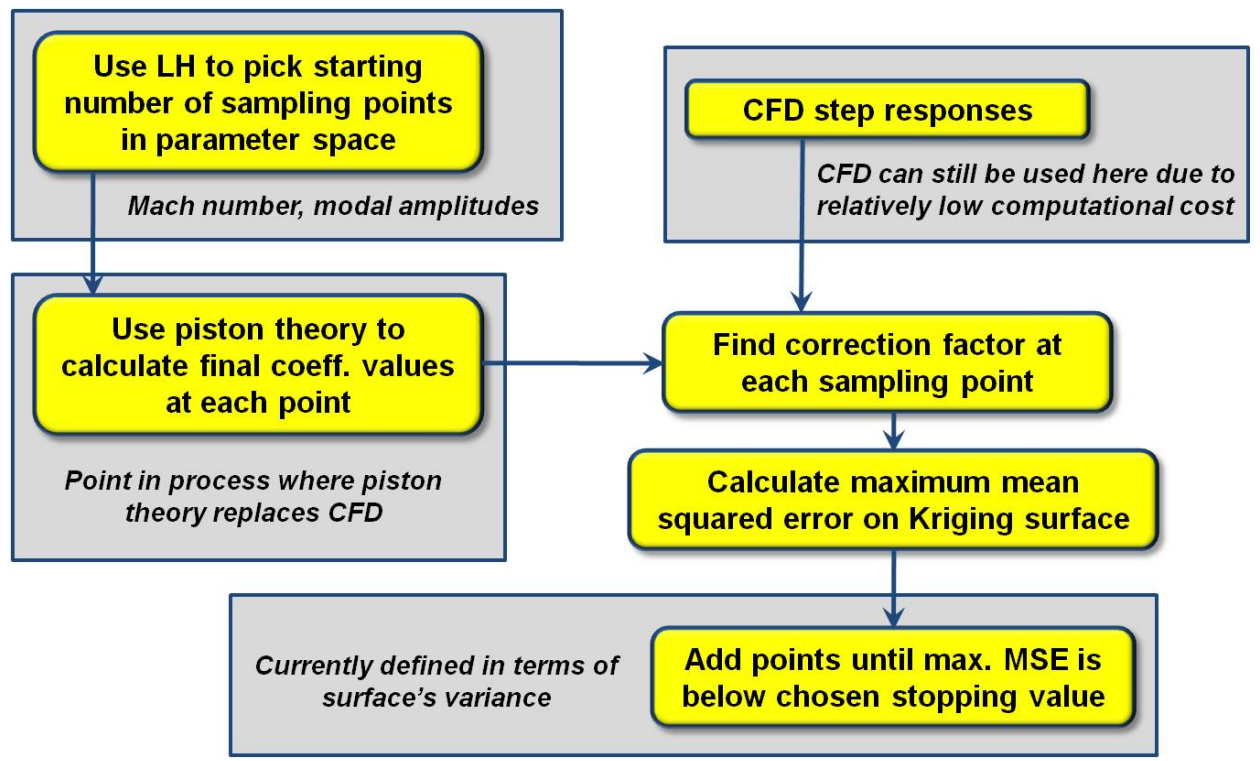

Figure 9. Error analysis: comparison of Kriging surface to function

A new sampling point is then added at the location of the maximum MSE. The process is repeated until the error has fallen below the designated stopping criterion. A benefit of this methodology is that the stopping criterion can be input by the user and is quantitative rather than qualitative. Currently, this criterion is defined in terms of the kriging surface's variance, as shown in Eq. 11, where $\max \left(s^{2}\right)$ is the maximum MSE on the surface:

$$
\max \left(s^{2}\right)<0.01 \sigma^{2}
$$


Figures 10 and 11 show a simple graphical example of this process. Five sampling points are at first selected to model the function $y=(x-2)(x-4)(x-9)$. The corresponding kriging fit and MSE plot are shown in Fig. 10. Then, the above process is applied, and the end result is shown in Fig. 11. Three more sampling points were added, and the function and kriging fit are indistinguishable in the plot.

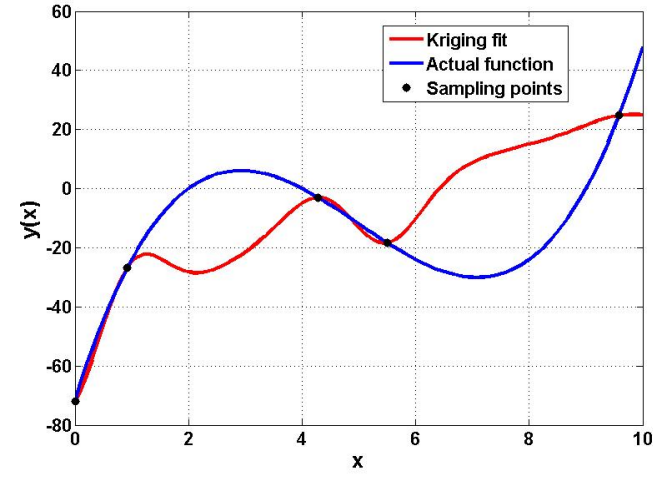

(a) Kriging fit

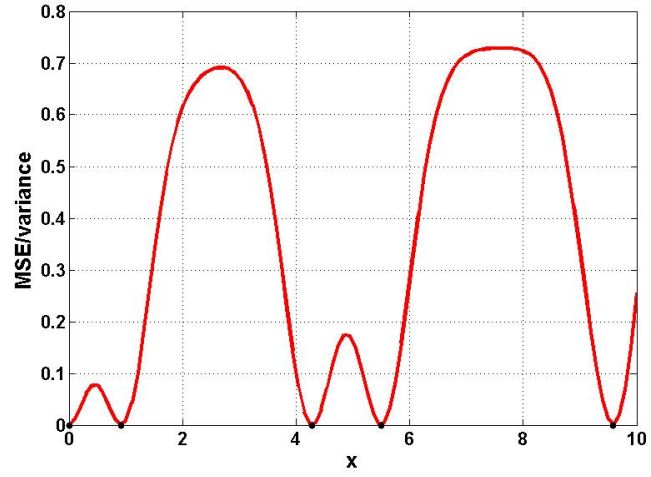

(b) Mean squared error

Figure 10. Kriging fit and MSE with initial sampling points

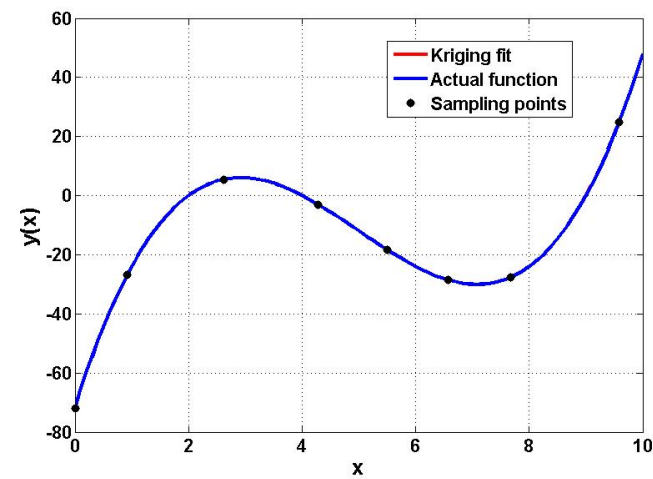

(a) Kriging fit

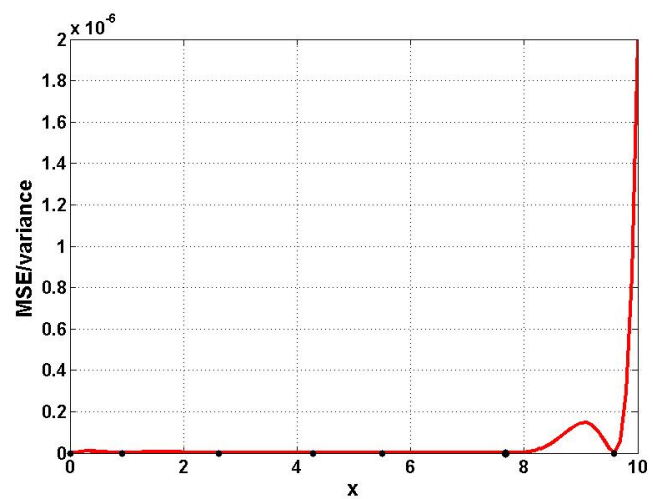

(b) Mean squared error

Figure 11. Kriging fit and MSE, error criterion satisfied

Using the stopping criterion detailed above, around 90 sampling points were necessary for the construction of the kriging surface of the two-dimensional half-diamond airfoil with Mach number and each of the three modal amplitudes as parameters. Latin hypercube test points were generated using MATLAB's lhsdesign subroutine ${ }^{20} 100,000$ iterations were used, and the sampling points with minimum correlation were chosen. Note that since the specific beginning sampling points will differ, the exact number of points necessary to achieve the stopping criterion was slightly different for each time the above process was repeated.

\section{B. Error of Function Compared to Truth Model}

Once the kriging surface has been constructed in such a way that it matches up well with the intended function, it is necessary to evaluate how well the function itself represents the truth model. Fig. 12 shows the overall process that has been implemented. As before, piston theory is utilized due to low computational expense.

The overall error is investigated by comparing ROM and truth model results over a large sample of test cases throughout the parameter space. To begin with, Latin hypercube sampling is used to pick points at 


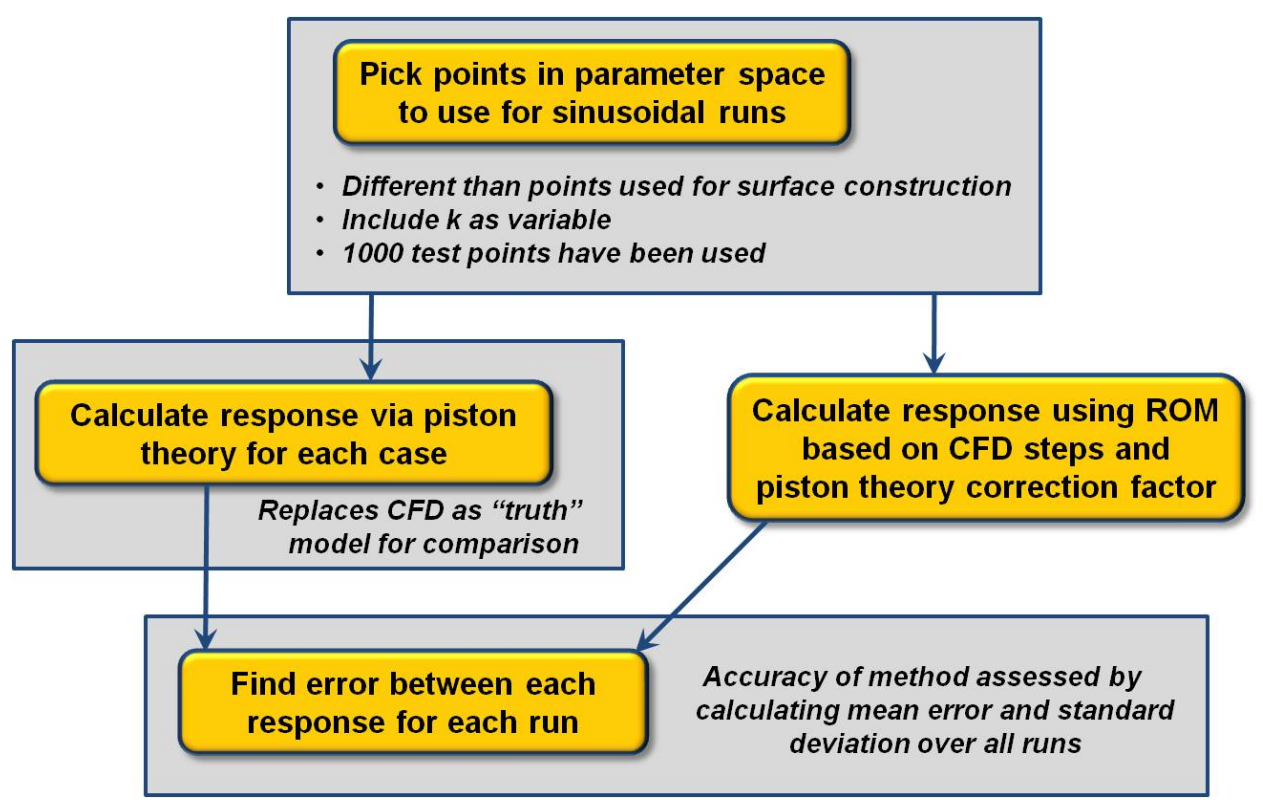

Figure 12. Error analysis: comparison of function to truth model

which to run sinusoidal test cases. In addition to Mach number and modal amplitudes, modal frequencies are also included as variables. These test points are in general different than the points used for kriging surface construction. For this study, 1,000 test points have been used. At each point, the the sinusoidal input response is calculated in two different ways: once using the ROM based on a CFD step response and piston theory correction factor and once using straight piston theory. The straight piston theory result here replaces the CFD model as the "truth" model for comparison. Finally, the error of ROM as compared to piston theory results is found for each run. The ROM methodology's accuracy is assessed by calculating the mean and standard deviation of the errors over all runs.

Table 3 shows the range of parameters used for this study as well as the specific parameters of the run which had the highest error (denoted as "Max. Error"). Fig. 13(a) shows a scatter plot of the errors of each run along with the mean and standard deviation superimposed on the plot, while Fig. 13(b) shows the comparison of the ROM, piston theory, and CFD results for the maximum error case.

Table 3. Error analysis parameters

\begin{tabular}{cccc}
\hline \hline Parameter & Min. Value & Max. Value & Max. Error \\
\hline$M$ & 5 & 9.5 & 8.56 \\
$d_{1}$ & $-60 \times$ & $60 \times$ & $-7.71 \times$ \\
$d_{2}$ & $-25 \times$ & $25 \times$ & $-13.02 \times$ \\
$d_{3}$ & $-25 \times$ & $25 \times$ & $13.13 \times$ \\
$k_{1}$ & 0.028 & 0.375 & 0.168 \\
$k_{2}$ & 0.028 & 0.375 & 0.044 \\
$k_{3}$ & 0.028 & 0.375 & 0.059 \\
\hline \hline
\end{tabular}

In general, the errors are relatively small. The overall mean of the errors, denoted by the solid red line in the figure, is $8.89 \%$ with a standard deviation of $4.44 \%$. The largest error, for which the ROM-truth model comparison is shown in Fig. 13(b), is just over 45\%. For this case, piston theory shows a closer agreement with the results than the ROM. For this geometry, piston theory has been shown to agree well with the CFD results in general. However, for more complex configurations, such as highly three-dimensional bodies, 


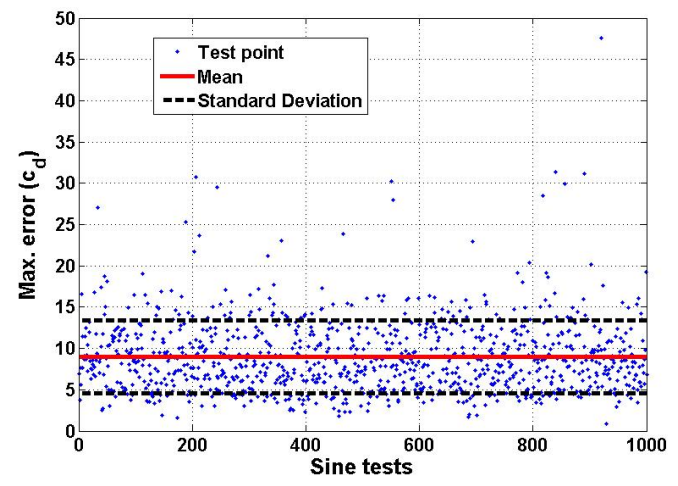

(a) Error analysis results

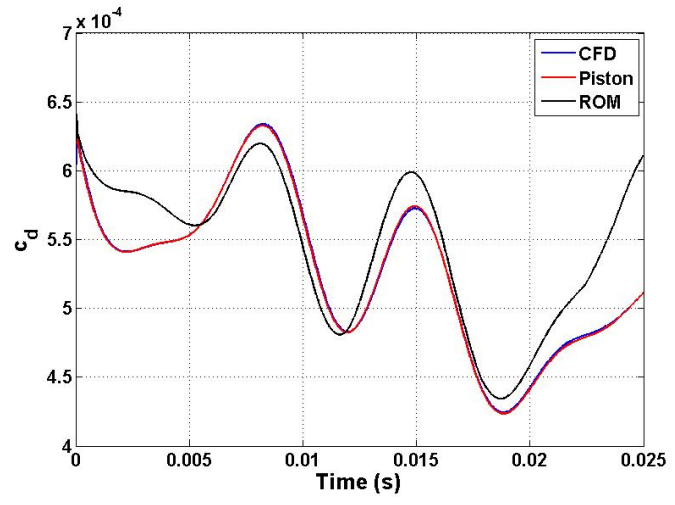

(b) Maximum error case

Figure 13. Results: comparison of function to truth model

piston theory will be expected to break down, whereas the ROM can be applied to any configuration.

The goal of this methodology is not to give an exact error that is expected to be incurred but rather a general picture of the error. For the more complex geometries, where piston theory will not be expected to give as accurate of results, the use of piston theory and/or other simplified models serve to give a picture of the general trends expected with the error, not a detailed accurate analysis of any specific errors seen.

\section{Concluding Remarks}

A convolution-based reduced-order modeling methodology for the unsteady aerodynamics of a hypersonic vehicle has been extended from capturing single-modal oscillation effects to multiple-modal oscillations. Studies have looked at the error incurred as functions of increasing reduced frequency. An error estimation methodology has been developed to investigate prior to model construction the general trends of the error expected to be encountered. This methodology includes a quantification of the number of sampling points necessary to construct the kriging surface as well as the use of simplified models to replace CFD results. For the two-dimensional half-diamond airfoil geometry used in this work, the major conclusions are as follows:

- Errors were shown to increase with increased reduced frequency. However, they remained relatively small over the range considered.

- The multi-modal correction factor was successfully applied to this configuration over a range of frequencies, modal oscillation amplitudes, and Mach numbers and showed improved agreement with CFD results over straight superposition of individual modal responses.

- Using the error estimation methodology, the mean error over 1,000 sample runs was found to be just under $9 \%$ with a standard deviation of slightly over $4 \%$.

Future work will consist of the application of the ROM to a three-dimensional rocket-type body where piston theory will not be expected to provide as accurate of results. Also, the error quantification results presented above are only valid for this specific configuration and do not provide general error bounds for the method. Further investigations will explore these bounds in terms of geometry and the ROM methodology's extension into other flight regimes.

\section{Acknowledgments}

Funds for the Michigan-AFRL Collaborative Center in Control Science (MACCCS) were made available from the Air Force Research Laboratory/Air Vehicles Directorate grant number FA 8650-07-2-3744. The program manager is Michael Bolender. 


\section{References}

${ }^{1}$ Glaz, B., Liu, L., Friedmann, P., Bain, J., and Sankar, L., "A Surrogate Based Approach to Reduced-Order Dynamic Stall Modeling," Proceedings of the 51st AIAA/ASME/ASCE/AHS/ASC Structures, Structural Dynamics, and Materials Conference, AIAA Paper No. 2010-3042, April 2010.

${ }^{2}$ Silva, W., "Recent Enhancements to the Development of CFD-Based Aeroelastic Reduced-Order Models," Proceedings of the 48th AIAA/ASME/ASCE/AHS/ASC Structures, Structural Dynamics, and Materials Conference, AIAA Paper No. 2007-2051, April 2007.

${ }^{3}$ Lind, R., Prazenica, R., Brenner, M., and Baldelli, D., "Identifying Parameter-Dependent Volterra Kernels to Predict Aeroelastic Instabilities," AIAA Journal, Vol. 43, No. 12, December 2005, pp. 2496-2502.

${ }^{4}$ Baldelli, D., Zeng, J., Lind, R., and Harris, C., "Flutter-Prediction Tool for Flight-Test-Based Aeroelastic ParameterVarying Models," Journal of Guidance, Control, and Dynamics, Vol. 32, No. 1, January-February 2009, pp. $158-171$.

${ }^{5}$ Prazenica, R., Reisenthel, P., Kurdila, A., and Brenner, M., "Volterra Kernel Extrapolation for Modeling Nonlinear Aeroelastic Systems at Novel Flight Conditions," Journal of Aircraft, Vol. 44, No. 1, January-February 2007, pp. 149-162.

${ }^{6}$ Omran, A. and Newman, B., "Piecewise Global Volterra Nonlinear Modeling and Characterization for Aircraft Dynamics," Journal of Guidance, Control, and Dynamics, Vol. 32, No. 3, May-June 2009, pp. 749-759.

${ }^{7}$ Skujins, T. and Cesnik, C., "Reduced-Order Modeling of Hypersonic Vehicle Unsteady Aerodynamics," Proceedings of the AIAA Atmospheric Flight Mechanics Conference, AIAA Paper No. 2010-8127, August 2010.

${ }^{8}$ Silva, W., "Discrete-Time Linear and Nonlinear Aerodynamic Impulse Responses for Efficient CFD Analyses," Ph.D. dissertation, College of William \& Mary, December 1997.

${ }^{9}$ Fung, Y., An Introduction to the Theory of Aeroelasticity, Dover Publications, Mineola, New York, 1993.

${ }^{10}$ Lophaven, S., Nielsen, H., and Sondergaard, J., "DACE: A MATLAB Kriging Toolbox, Version 2.0," Tech. Rep. IMMTR-2002-12, Informatics and Mathematical Modelling, Denmark, August 1, 2002.

${ }^{11}$ Sacks, J., Welch, W., Mitchell, T., and Wynn, H., "Design and Analysis of Computer Experiments," Statistical Science, Vol. 4, No. 4, 1989, pp. 409-435.

${ }^{12}$ Rumsey, C. and Biedron, R., "CFL3D User's Manual, Version 5.0, Second Edition," Tech. Rep. Hampton, VA, NASA Langley Research Center, September 1997 (last updated August 2009).

${ }^{13}$ Anon., "Documentation for ANSYS ICEM CFD 12.0," Tech. rep., ANSYS, Canonsburg, Pennsylvania, 2009.

${ }^{14}$ Cioppa, T. and Lucas, T., "Efficient Nearly Orthogonal and Space-Filling Latin Hypercubes," Technometrics, Vol. 49, No. 1, February 2007, pp. 45-55.

${ }^{15}$ Jones, R., Schonlau, M., and Welch, W., "Efficient Global Optimization of Expensive Black-Box Functions," Journal of Global Optimization, Vol. 13, No. 4, 1998, pp. 455-492.

${ }^{16}$ Glaz, B., Friedmann, P., and Liu, L., "Efficient Global Optimization of Helicopter Rotor Blades for Vibration Reduction in Forward Flight," Proceedings of the 11th AIAA/ISSMO Multidisciplinary Analysis and Optimization Conference, AIAA Paper No. 2006-6997, September 2006.

${ }^{17}$ Ashley, H. and Zartarian, G., "Piston Theory - A New Aerodynamic Tool for the Aeroelastician," Journal of the Aeronautical Sciences, Vol. 23, No. 12, 1956, pp. 1109-1118.

${ }^{18}$ Lighthill, M., "Oscillating Airfoils at High Mach Number," Journal of the Aeronautical Sciences, Vol. 20, No. 6, 1953, pp. 402-406.

${ }^{19} \mathrm{McNamara}$, J. and Friedmann, P., "Aeroelastic and Aerothermoelastic Analysis of Hypersonic Vehicles: Current Status and Future Trends," Proceedings of the 48th AIAA/ASME/ASCE/AHS/ASC Structures, Structural Dynamics, and Materials Conference, AIAA Paper No. 2007-2013, April 2007.

${ }^{20}$ Anon., "MATLAB Version 7.10.0 User Guide," Tech. rep., The MathWorks, Inc., Natick, MA, 2010. 